\title{
Shocking degeneration
}

\author{
Rainer Benndorf \& Michael J Welsh \\ Mammalian small heat-shock proteins are suspected to have a role in neuromuscular function. Two new studies \\ provide evidence for the association of mutations in two of these proteins, HSP22 and HSP27, with human \\ neuromuscular disorders.
}

In this issue, Joy Irobi and colleagues ${ }^{1}$ and Oleg Evgrafov and colleagues ${ }^{2}$ report the association of missense mutations in two small heat-shock proteins (sHSPs), HSP22 and HSP27, with the human neuromuscular disorders axonal Charcot-Marie-Tooth disease and distal hereditary motor neuropathy. These findings, together with previous reports of myopathy-related mutations in another sHSP, $\alpha \mathrm{B}$-crystallin ${ }^{3,4}$, and the fact that mutations in $\alpha \mathrm{B}$-crystallin ${ }^{3,5}$ and in $\alpha \mathrm{A}$-crystallin ${ }^{6-8}$ have been associated with congenital cataracts, change both our understanding of the functions of sHSPs and future approaches to researching neuromuscular disorders.

Table 1 lists human sHSPs (and their synonyms), the positions of the mutations and the associated diseases. Most of the neuromyopathy-related mutations are positioned in the $\alpha$-crystallin domain, the most conserved part of these proteins, and three are in the adjacent highly variable C-terminal tails. Mutations associated with congenital cataracts have also been found in the N-terminal parts of sHSPs. There is a homologous mutational hot spot in three proteins $(\alpha \mathrm{A}$ crystallin, $\alpha \mathrm{B}$-crystallin and HSP22) that is associated with disease.

\section{Hidden importance of sHSPs}

Proteins that share the $\alpha$-crystallin domain, a sequence of $\sim 85$ amino acid residues in the Cterminal region, belong to the superfamily of sHSPs, whether or not their expression is

Rainer Benndorf and Michael J. Welsh are in the Department of Cell and Developmental Biology at the University of Michigan Medical School, Ann Arbor, Michigan, USA.

e-mail: rbenndo@umich.edu heat-inducible. The identification of mammalian sHSPs was a century-long effort. The $\alpha$-crystallins were first identified in 1894 (ref. 9), but only in 2003 was the list of the ten mammalian sHSPs completed with the recognition of sperm outer dense fiber protein as HSPB10 (refs. 10,11). In the interim, many biologists accumulated considerable data on the structure and functions of sHSPs.

In spite of this effort, today's knowledge of sHSPs still resembles more a heap of puzzle pieces than a whole picture. We know that sHSPs are part of signal transduction cascades and tend to form oligomeric complexes. Some sHSPs (HSP27, HSP22, HSP20, $\alpha \mathrm{A}$-crystallin, $\alpha \mathrm{B}$-crystallin) have chaper-

one-like properties in vitro, and in vivo they support cell survival under stress conditions (e.g., heat, toxic metals, anticancer drugs, oxidative stress) by inhibiting apoptosis. It is widely believed that this is their original function. Other, more specific roles of sHSPs, such as stabilizing cytoskeletal systems (HSP27, $\alpha B$-crystallin) or activating myotonic dystrophy protein kinase may be derived from this property. sHSPs are also involved in cytoskeletal functions such as cell motility and migration, and they modulate contraction and relaxation of muscles. sHSP function is related to intermediate filaments, as shown by the facts that mutations in HSP27 and neurofilament light chain result

Table 1 Identified mutations in human sHSPs and associated diseases

\begin{tabular}{|c|c|c|c|c|}
\hline sHSPa & Mutation & Affected sHSP region & Diagnosis & Reference \\
\hline \multirow[t]{5}{*}{ HSP27 } & 379C>T; R127W (missense) & $\alpha$-crystallin domain & $\mathrm{dHMN}$ & 2 \\
\hline & 404C>T; S135F (missense) & $\alpha$-crystallin domain & CMT2, dHMN & 2 \\
\hline & 406C>T; R136W (missense) & $\alpha$-crystallin domain & СMT2 & 2 \\
\hline & 452C>T; T151I (missense) & $\alpha$-crystallin domain & dHMN & 2 \\
\hline & 545C>T; P182L (missense) & C-terminal tail & dHMN & 2 \\
\hline \multirow[t]{2}{*}{ HSP22 } & $421 \mathrm{~A}>\mathrm{G} ; \mathrm{K} 141 \mathrm{E}$ (missense) $^{\mathrm{b}}$ & $\alpha$-crystallin domain & dHMN type II & 1 \\
\hline & $423 \mathrm{G}>\mathrm{C} ; \mathrm{K} 141 \mathrm{~N}$ (missense) $^{\mathrm{b}}$ & $\alpha$-crystallin domain & dHMN type II & 1 \\
\hline \multirow[t]{4}{*}{$\alpha B$-crystallin } & $358 \mathrm{~A}>\mathrm{G} ; \mathrm{R} 120 \mathrm{G}$ (missense) $^{\mathrm{b}}$ & $\alpha$-crystallin domain & $\mathrm{DRM}+\mathrm{CC}$ & 3 \\
\hline & 464delCT (aberrant protein) & C-terminal tail & MM & 4 \\
\hline & 451C>T; Q151X (truncation) & C-terminal tail & MM & 4 \\
\hline & 450delA (aberrant protein) & C-terminal tail & posterior polar CC & 5 \\
\hline \multirow[t]{3}{*}{$\alpha A$-crystallin } & $413 \mathrm{C}>\mathrm{T} ; \mathrm{R} 116 \mathrm{C}$ (missense) $^{\mathrm{b}}$ & $\alpha$-crystallin domain & $\mathrm{CC}$ & 6 \\
\hline & 145C>T; R49C (missense) & $\mathrm{N}$-terminal domain & $\mathrm{CC}$ & 8 \\
\hline & 27G>A; W9X (truncation) & $\mathrm{N}$-terminal domain & $\mathrm{CC}$ & 7 \\
\hline
\end{tabular}

aSynonyms for all ten mammalian SHSPs: HSP27 (HSPB1, HSP25); myotonic dystrophy protein kinase binding protein (MKBP, HSPB2); HSPB3, $\alpha$ A-crystallin (HSPB4); $\alpha$ B-crystallin (HSPB5); HSP2O (HSPB6); cvHSP (HSPB7); HSP22 (HSPB8, H11, E2IG1); HSPB9; sperm outer dense fiber protein 1 (ODFP1, HSPB10). bMutations in homologous positions. dHMN, distal hereditary motor neuropathy; CMT2, Charcot-Marie-Tooth disease type 2; DRM, desmin-related myopathy; MM, myofibrillar myopathy; CC, congenital cataract. 
in similar disease phenotypes ${ }^{1}$ and that the R120G $\alpha$ B-crystallin mutation results in aggregation of desmin filaments ${ }^{3}$. Most sHSPs ( $\alpha$ B-crystallin, HSP27, HSP22, HSP20, MKBP, HSPB3, cvHSP) are particularly abundant in muscles and heart, where they may comprise $3 \%$ or more of total protein. At least three sHSPs ( $\alpha \mathrm{B}$-crystallin, HSP27, HSP22) are abundant in different nerve cells, and $\alpha \mathrm{B}$-crystallin and HSP27 accumulate in individuals with various neurodegenerative disorders, including Alzheimer, Parkinson and Alexander diseases and multiple sclerosis. Thus, that mutations in sHSPs would cause neuromuscular disorders is not entirely unexpected. More baffling is the decade-long ignorance of sHSPs by neuromuscular researchers.

Although a number of interacting partners have been identified, the most obvious interactions involving sHSPs occur with each other, resulting in formation of homo- and hetero-oligomeric complexes. Indeed, formation of complexes with a wide range of molecular masses is the most pronounced, though least understood, characteristic of sHSPs. These complexes are dynamic structures, with subunits exchanging rapidly. This situation may contribute to the difficulty of understanding the cellular function of sHSPs. Whatever their precise role is at the molecular level, the properties of the ten sHSPs are probably not identical. For example, the sequences of the C-terminal tails of the ten proteins, which are distinguished by their high conformational flexibility, are very different and probably confer unique properties on each sHSP. The fact that mutations in different sHSPs are associated with similar, but not identical, diseases support this idea of distinct roles for these proteins. Moreover, the relatively late onset of some neuromuscular diseases, which suggests an accumulative tissue damage pattern, indicates a more indirect, perhaps protective role of the wild-type sHSPs rather than an immediate role in proper tissue function.

\section{What's next?}

What is the degree of functional redundancy and difference among the ten mammalian sHSPs? Can sHSPs other than HSP27, $\alpha \mathrm{B}-$ crystallin, $\alpha \mathrm{A}$-crystallin and HSP20 protect cells from stress? How does the composition of sHSP complexes vary in different cell types? Do complexes vary during development or in response to physiological signals? Is the composition of complexes regulated, thus allowing a fine-tuning of the functions of sHSP complexes? What is the functional importance of various hetero-oligomeric complexes? Indeed, the different expression patterns of sHSPs in different organs and tissues and the idea that probably not all sHSPs are stress-inducible suggest that the composition and functions of sHSP complexes may be cell- and stress-specific.

Regarding the heterogeneous group of motor neuropathies and muscular dystrophies of unknown cause, the identification of more sHSP mutations can be expected. Although poor understanding of sHSP functions will impede development of therapeutic strategies in the near future, there is reason to be optimistic that much will be learned about the molecular and cellular role of sHSPs in the coming decade, as the importance of this protein superfamily becomes more widely recognized.

1. Irobi, J. et al. Nat. Genet. 36, 597-601 (2004).

2. Evgrafov, O.V. et al. Nat. Genet. 36, 602-606 (2004).

3. Vicart, P. et al. Nat. Genet. 20, 92-95 (1998).

4. Selcen, D. \& Engel, A.G. Ann. Neurol. 54, 804-810 (2003).

5. Berry, V. et al. Am. J. Hum. Genet. 69, 1141-1145 (2001).

6. Litt, M. et al. Hum. Mol. Genet. 7, 471-474 (1998).

7. Pras, E. et al. Invest. Ophthalmol. Vis. Sci. 41, 3511-3515 (2000)

8. Mackay, D.S., Andley, U.P. \& Shiels, A. Eur. J. Hum Genet. 11, 784-793 (2003).

9. Mörner, C.T. Hoppe Seylers Z. Physiol. Chem. 18, 61-106 (1894).

10. Kappe, G. et al. Cell Stress Chaperones 8, 53-61 (2003).

11. Fontaine, J.M., Rest, J.S., Welsh, M.J. \& Benndorf, R. Cell Stress Chaperones 8, 62-69 (2003).

\title{
Sex and the genome
}

\author{
Valerie Reinke
}

The genetic composition of sex chromosomes has been deciphered, in part, through large-scale gene expression studies in different species. A new study in mice adds a missing piece of the puzzle: the composition of the $X$ chromosome in mammals is influenced by inactivation of the sex chromosomes during male meiosis.

Sex complicates many aspects of life but is sufficiently beneficial that virtually every eukaryotic species uses it. An especially sticky consequence is that an unequal complement of sex-determining chromosomes exists between the two sexes. One sex, typically female, carries two X chromosomes, and the other sex, usually male, has a single $\mathrm{X}$ chromosome (accompanied by a $\mathrm{Y}$ chromosome in some, but not all, species). This inequality puts the sex chromosomes in an evolutionarily vulnerable position.

Valerie Reinke is in the Department of Genetics, Yale University School of Medicine, New Haven, Connecticut, USA.

e-mail: valerie.reinke@yale.edu
Two hypotheses propose different outcomes for sex-linked genes that are preferentially expressed in one sex over the other. Rice's hypothesis ${ }^{1}$ states that, because males carry a single $\mathrm{X}$ chromosome, any recessive allele arising on the $\mathrm{X}$ that gives males a reproductive advantage is immediately available for positive selection. Conversely, any negative effects of that allele in females are masked by the presence of a second X chromosome. Thus, genes expressed preferentially in males are more likely to be present on the $\mathrm{X}$ chromosome. An alternate hypothesis states that the $\mathrm{X}$ should be feminized because the $\mathrm{X}$ chromosome is present in females 50\% more frequently than in males, providing evolution with more opportunity to act on genes benefiting females. Thus, genes with female-biased expression should reside on the $\mathrm{X}$ chromosome. So which hypothesis is correct, or are the two hypotheses mutually compatible? In this issue, Pavel Khil and colleagues ${ }^{2}$ look at sex-biased expression profiles in mice and show that, as for many relationships, the answer involves a compromise.

\section{On again, off again}

Khil et al. adopted a computational approach using publicly available mouse expressedsequence tag and microarray databases to define genes expressed preferentially in various somatic and gonadal tissues. They then 